\title{
Analysis approach of turnout crossing performance by field measurements and finite element modeling
}

L.Xin, V.L.Markine

Delft University of Technology, Delft, The Netherlands

\section{I.Y. Shevtsov}

ProRail, Utrecht, The Netherlands

ABSTRACT: The procedure for analyzing turnout crossing performance is developed in this paper. The experimental and numerical analysis are both conducted to evaluate the dynamic behavior of the crossing and to further improve the crossing performance. Geometry and acceleration measurements are performed on common single turnouts in the Dutch railway network for analyzing the measured crossing performance and providing the input for numerical modeling. Meanwhile, a three-dimensional finite element model of a whole wheelset rolling over the crossing has been developed. The wing rail and crossing nose geometry as well as the wheel geometry have been used in the model. The numerical responses of the model comprise the dynamic contact forces between the wheelset and the crossing, displacements of the wheelset and crossing as well as the local contact stress and strain distributions, by which the crossing performance is evaluated and the fatigue life of the crossing is predicted. By this approach further improvement of the crossing nose and wing rail geometry can be realized. 


\section{INTRODUCTION}

\subsection{Turnout crossing}

Turnouts are important elements of the railway infrastructure as they provide guidance to the traffic. However, they are also vulnerable elements of railways that cause most of the operation disturbance. A turnout consists of a switch panel, a closure panel and a crossing panel (Figure 1). For the crossing panel the rail geometry is discontinuous, where space between the wing rail and crossing nose exists. Therefore, when the vehicles pass the turnout, high impact forces are generated on the crossing nose or on the wing rail. Compared with the normal (tangent) track, the wheel forces in the crossing can be up to two to four times higher [1]. Moreover, since the shape of the crossing profiles is gradually changing to the normal rail, the small width and radius of the crossing nose make it the weakest point in the crossing structure, since the high forces can lead to high stresses in the rail. Therefore, the high impacts forces and the variable crossing geometry together result in severe damage in the crossing.

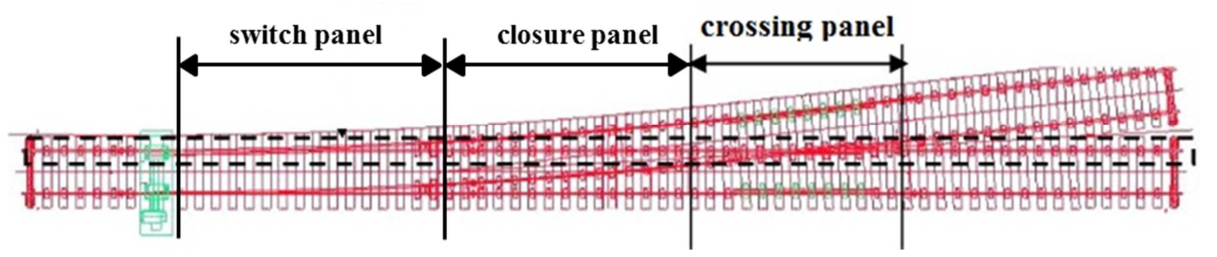

Figure 1. General layout of turnout.

In the Netherlands, turnout crossing is one of the main causes of railway disturbance and an increased damage to the crossings is observed. According to statistics in the Netherlands [2], where there are more than 8,000 switches and crossings in operation, almost 400 crossings replaced per year and two crossings per week are urgently to be repaired. The replacement budget has reached 6.4 million per year. Obviously, solutions for the crossing related problems are urgently needed.

\subsection{Influential parameters}

There are several parameters that may influence the wheel/crossing interaction, among which the geometry and material properties of the wheel and crossing play important roles. Several research studies have been conducted on the effect of the crossing geometry, such as the optimization of the crossing geometry, which proves that the slightly changed geometry can lead to great improvement or degradation in wheel/crossing interaction $[3,4]$. It should be noted that regarding to the rail geometry, except for the crossing nose geometry that has been discussed above, the geometrical irregularities before and after the crossing panel could also have a great influence on the wheel/crossing impact. It was observed in the field in the Netherlands that the long wave irregularity of the rail resulted in fast degradation of the crossing geometry and ultimately damage of crossing.

The material properties are closely related to the amount of wear and rolling contact fatigue (RCF) of the wheel and crossing. Many researches related to crossing material have been conducted. For example, three crossing nose materials were studied by the multi-scale FE approach, in which the crack development and growth of the three materials were obtained and compared [5]. Experimental investigations of fatigue properties were conducted of a cast austenitic manganese steel that was used in railway turnouts and crossings [6]. In [7] a crossing nose model with welding defect that generating a layer of martensite in the material was simulated and analyzed.

Moreover, different loading conditions, vehicle velocities, friction coefficient [8] and supporting stiffness [2] etc. may also result in various wheel/crossing interaction. For example, the dynamic behaviour of the turnout crossing, including the variation of elastic track properties was studied in [2,9] using the two-dimensional (2-D) finite element software DARTS_NL. In [2] the contact forces in the trailing travelling direction of a train with three velocities were simulate. The results showed that high impact was generated on the wing rail in all three cases. 
Damage on turnout influenced by a stochastic spread in traffic parameters such as wheel profile and friction coefficient was analyzed in [10].

\subsection{Analysis procedure}

In order to analyze and improve the crossing performance, research studies should concern both realistic crossing performance in the field measurements and the numerical modeling which can take difference parameters into account. The measurements show the wheel/crossing interaction in the field and provide the input for numerical analysis. Meanwhile the numerical tools are used to analyze and further improve the crossing performance, for example by tuning the geometry or the elasticity of the rail components.

Therefore, the analysis procedure in this paper can be divided into two parts: the experimental and numerical analysis of the crossing. Measurements of the crossing were conducted within the railway network in the Netherlands including different conditions. The numerical analysis is performed by the three-dimensional (3-D) explicit finite element modeling, which was developed to simulate the dynamic response of a whole wheelset while passing a crossing. Using this model the dynamic contact forces between the wheelset and the crossing, displacements of the wheelset and crossing as well as the local contact stress distributions in the rail can be obtained.

\section{EXPERIMENTAL RESEARCHES ON TURNOUT CROSSING}

As mentioned above turnout is one of the main causes of railway disturbance. Therefore many measurements of turnout crossing were conducted to investigate the dynamic behavior of the crossing. Field observations, geometry and acceleration/displacement measurements of the dynamic behavior of the crossing during wheel passage form the basics of the experimental researches. Several researches can be then performed, such as diagnose of a problematic crossing, quality assessment of welding and grinding of the crossing nose as well as the longterm monitoring of a particular crossing. All these experimental researches help understand and provide the possibilities of improvement of the crossing performance.

\subsection{Geometry measurements}

The geometry measurements of the crossing were performed by a laser-based device called Calipri. The device can measure cross-sectional geometry including railway wheels and rails (Figure 2a). In this study a number of profiles along the crossing are measured. The interval of adjacent measured rail profiles was $50 \mathrm{~mm}$ from around $300 \mathrm{~mm}$ before the nose point to 1050 $\mathrm{mm}$ after the nose point.

The geometry can be used to analyze the conditions of a crossing such as the amount of plastic deformation and the location of the problematic geometry. Also by the measured geometry the dynamic behavior can be related to geometry, which enables to assess how the geometry affected the crossing performance, such as measuring the crossing geometry before after the welding and grinding. The measured profiles are also used to build up the numerical model of the crossing (Figure 2b), for different analysis such as model validation, problem diagnose of a given crossing as well as the geometrical improvement of the crossing. 
a

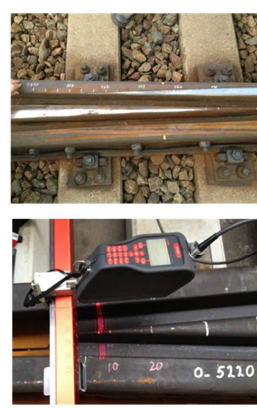

b
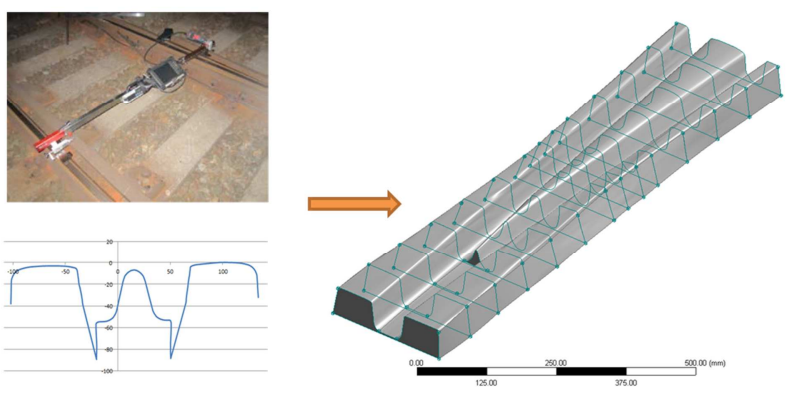

Figure 2. (a) Rail geometry measurement, (b) Crossing model with the imported profiles

\subsection{Acceleration/ displacement measurements}

The measurement device ESAH-M for analyzing wheel-rail interaction in the crossing consists of a triaxial acceleration sensor to be placed on the crossing nose, two inductive sensors to be installed on the rail for wheel velocity measurements and a sleeper displacement sensor. Based on the measured velocity of the passing wheels and the distance between the velocity sensor and the beginning of the crossing nose, the magnitude and location of the maximum accelerations for each passing wheel is determined. The sampling frequency of the accelerometer in the measurements is $10 \mathrm{kHz}$. Details about the measurement process can be found in [11] and [12].

As an example, the measurements performed on a 1:15 crossing in the Dutch railway network were selected. Figure 3 a shows the crossing acceleration measurement setup. The passing train had the average velocity of $135 \mathrm{~km} / \mathrm{h}$. Figure $3 \mathrm{~b}$ shows the contact positions and the maximum accelerations of the crossing nose when trains were passing. From this figure the impact mostly occurs at a position of $500 \mathrm{~mm}$ to $650 \mathrm{~mm}$ from the nose point. Based on these data, the finite element model was developed focusing on the parts, which are affected by the impact the most. Figure $3 \mathrm{c}$ plots the measured maximum vertical accelerations of one train, in which the maximum acceleration of each wheelset is obtained. The same approach can be used to assess the wheel quality, which means that the wheel with too high or low acceleration may be regarded as bad wheel.

a

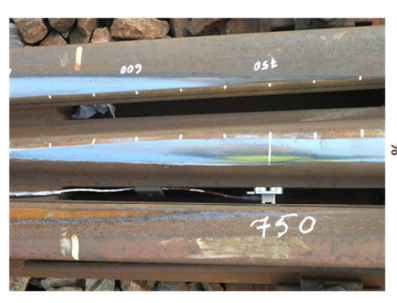

b

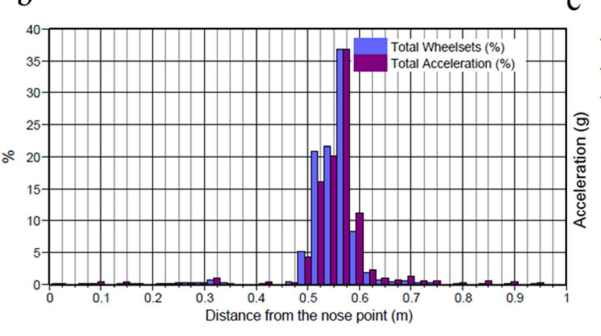

c

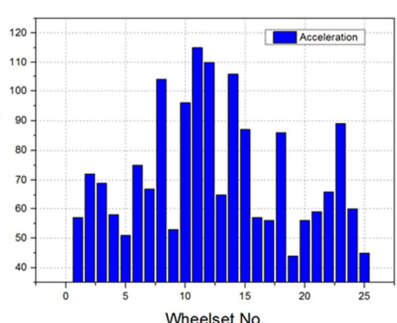

Figure 3. (a) Instrumented crossing, accelerations of all trains (b) and one train (c)

\section{NUMERICAL ANALYSIS OF TURNOUT CROSSING}

In this paper, a three dimensional (3-D) explicit finite element (FE) model (Figure 4a) with the implementation of elastic-plastic nonlinear kinematic hardening material is developed to simulate the dynamic response of a whole wheelset while it is passing a crossing $[8,9]$. The model consists a crossing section of $4540 \mathrm{~mm}$ long and the $\mathrm{S} 1002$ unworn wheel profile is used. The turnout modelled here is the curved left-handed turnout with the radius of $725 \mathrm{~m}$ and the crossing angle of 1:15. The wheelset is placed at a position of $376 \mathrm{~mm}$ before the crossing nose, which will roll over the crossing during the simulation. All the components in the model 
including railpads, sleepers and ballast are modelled using solid elements, which enables performing the stress and strain analysis. Fine mesh is used in the contact regions, i.e. the crossing nose, railhead and wheel tread (Figure 4b). Settings of the model will be discussed below in details.

a

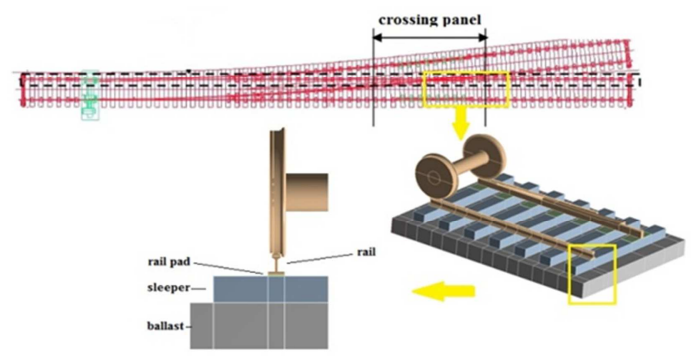

$\mathrm{b}$

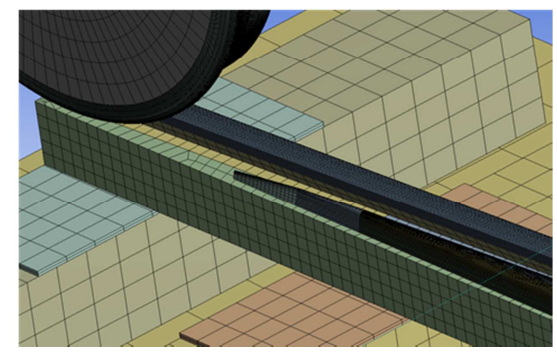

Figure 4. (a) schematic of the finite element model, (b) mesh of the wheel, crossing and the substructures [13].

\subsection{Geometry and material properties}

According to the purpose of studies the crossing model can be built in different ways. Generally the crossing model can be developed using four main cross-sections as they are defined in the drawings from the manufacturer (Figure 5a). These four cross-sections are located on the distances of $10 \mathrm{a}(\mathrm{mm}), 10 \mathrm{a}(\mathrm{mm})$ and $50 \mathrm{a}(\mathrm{mm})$ from each other, where the ' $\mathrm{a}$ ' is equal to the crossing angle of the turnout (e.g. for 1:15 turnout $a=15$ ). Except for the drawing as the reference case, crossing model can be also based on the geometry measurements by Calipri as mentioned in Section 2.1. It enables the numerical analysis of any particular turnout crossing in the field, mostly with worn geometry.

Another way can be importing geometry from other commercial software. For example, the dynamic response of the wheelset/crossing interaction conducted by multi-body system (MBS) model and finite element model is conduced and compared in [14]. In this case the crossing geometry should be the same. Therefore, the crossing and wing rail in the model are built by the profiles obtained from MBS model, which starting from $425 \mathrm{~mm}$ before the nose point to $1050 \mathrm{~mm}$ after nose point. These profiles are imported into FE model as 'sketches' onto a series of planes, which define the locations of the profiles (Figure $5 \mathrm{~b}$ ). They are then connected by 'skin' operation in ANSYS Workbench DesignModeler to form the 3D geometry. It also enables any imported geometry to be checked in FE model, such as the optimized geometry.

a

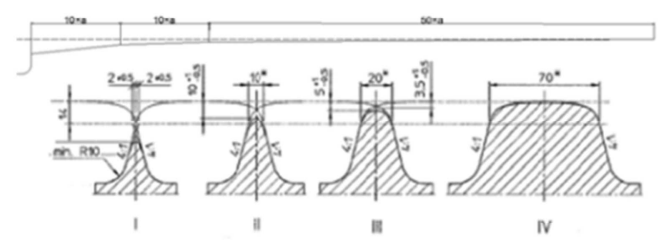

b

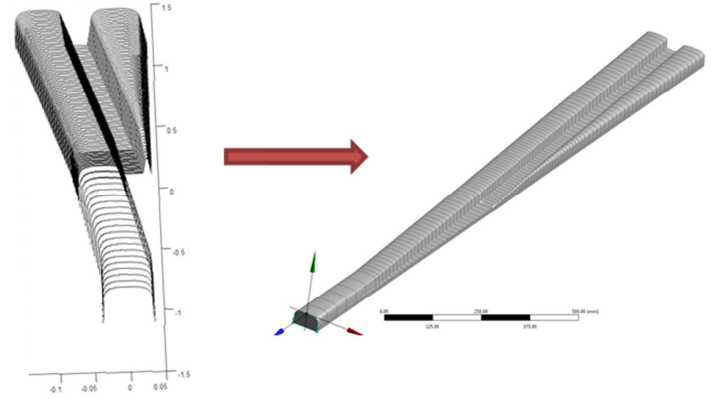

Figure 5. (a) crossing nose layout in the drawing, (b) imported geometry from MBS model

Since heavy repeated impact load is applied on the crossing, large plastic deformation is accumulated. Therefore, crossing in this paper is modelled by an elastic-plastic isotropic and kinematic hardening material model, which is based on Lemaitre and Chaboche [1990] material 
model. It is well suited to model nonlinear isotropic and kinematic plasticity and accounts for cyclic hardening and ratcheting. The size of the surface increase as function of the equivalent plastic strain, $\varepsilon^{\mathrm{p}}$ :

$$
\sigma_{\mathrm{y}}=\sigma_{\mathrm{y} 0}+\mathrm{H} \varepsilon^{\mathrm{p}}(3)
$$

Where $\sigma_{\mathrm{y} 0}$ is the initial stress; $\mathrm{H}$ is the isotropic hardening modulus.

The rate of evolution of the kinematic component is a function of the plastic strain rate:

$$
\dot{\alpha}=[\mathrm{Cn}-\gamma \alpha] \dot{\varepsilon}^{p}
$$

Where, $\alpha$ is the back stress, and $\mathrm{n}$ is the flow direction. $\mathrm{C}$ and $\gamma$ are the parameters for the back stress. The term, $\gamma \alpha \dot{\varepsilon}^{p}$, introduce the nonlinearity into the evolution law. The parameters of the material model can be found in [15]. The material parameters for elastic behaviour, isotropic hardening $\mathrm{H}$ and kinematic hardening $\mathrm{C}$ and $\gamma$ are listed in Table 1.

\begin{tabular}{|c|c|c|c|c|}
\hline Parameters (unit) & Value & \multicolumn{2}{|l|}{ Parameters (unit) } & Value \\
\hline Initial yield stress, $\sigma_{\mathrm{yo}},(\mathrm{MPa})$ & 500 & \multirow{3}{*}{ Young's Modulus (Pa) } & Railpad & $2.40 \mathrm{e}+8$ \\
\hline $\begin{array}{l}\text { Isotropic plastic hardening modulus, } \mathrm{H} \text {, } \\
(\mathrm{GPa})\end{array}$ & 20 & & Sleeper & $3.00 \mathrm{E}+10$ \\
\hline Kinematic hardening modulus, $\mathrm{C},(\mathrm{Gpa})$ & 13.2 & & Ballast & $1.34 \mathrm{E}+8$ \\
\hline Kinematic hardening parameter, $\gamma$ & 3.12 & \multirow{3}{*}{ Poisson's ratio } & Railpad & 0.47 \\
\hline Young's modulus (GPa) & 200 & & Sleeper & 0.18 \\
\hline Poisson's ratio & 0.30 & & Ballast & 0.20 \\
\hline Mass $(\mathrm{kg} / \mathrm{m} 3)$ & 7850 & \multirow{2}{*}{\multicolumn{2}{|c|}{$\begin{array}{l}\text { Tensile fatigue ductility } \\
\text { coefficients, } \varepsilon_{\mathrm{f}}^{\prime}\end{array}$}} & \\
\hline Tensile fatigue strength, $\sigma_{\mathrm{f}}^{\prime}(\mathrm{MPa})$ & 936 & & & 0.103 \\
\hline $\begin{array}{l}\text { Shear fatigue strength coefficients, } \tau_{\mathrm{f}}^{\prime} \\
(\mathrm{MPa})\end{array}$ & 468 & \multicolumn{2}{|c|}{ Shear fatigue ductility coefficients, $\gamma_{\mathrm{f}}^{\prime}$} & 0.1545 \\
\hline $\mathrm{b}$ & -0.089 & \multicolumn{2}{|l|}{$\mathrm{C}$} & -0.559 \\
\hline
\end{tabular}

Table 1. Material properties [13].

\subsection{Analysis procedure}

During the simulation, the wheelset moves along the crossing with the translational velocity of $140 \mathrm{~km} / \mathrm{h}$ and the angular velocity of $84.54 \mathrm{rad} / \mathrm{s}$. Initially, the wheels come in contact with the rails due to application of the gravity force and the axle load. In order to minimize the initial system vibration, the implicit preload procedure was used. After this stabilization stage the wheel set starts rolling. During the simulation, the inner wheel of the wheelset first rolls on the wing rail and then comes in contact with the crossing nose that generates an impact between the wheel and rail. Finally, the contact point transfers from the wing rail to the crossing nose and the wheel continues to roll on the through rail. The simulation time of $50 \mathrm{~ms}$ has been chosen which is sufficient for analysis of the wheel/rail impact. The friction coefficient of 0.2 has been used in the simulations.

The normal contact in this model is treated using penalty method and the contact force is proportional to the penetration depth and the interface stiffness to resist the penetration. The tangential force that consists of lateral contact force and longitudinal contact force, is calculated as the friction force, which is based on Coulomb friction.

The implicit-explicit switch method is used in these simulations, where the implicit method is adopted for the preload stage. After the preload stage the simulation is performed with explicit method. The explicit time integration is more accurate and efficient for simulations involving non-linear material behaviour and complex contact, which is closely relevant to the wheel/rail contact simulation. However, it requires tiny time step to get stable, so it is usually suitable for short duration event. The time step in this case was $3 \mathrm{e}-7 \mathrm{~s}$ and remained the same in the whole calculation. 


\section{RESULTS}

By the FE modeling the dynamic responses of the system such as the contact forces between the wheel and the crossing, crossing nose displacements and accelerations, stresses in rail material as well as in sleepers and ballast are obtained. The stress and strain distributions on the crossing indicate the critical position to damage that is compared with the field measurements. With the stress/strain results, fatigue life analysis of the crossing is performed. The fatigue life to crack initiation can be calculated according to certain approaches, which in this study a combined energy-density based and critical plane approach is used. Details about the procedure and results can be found in [13].

\subsection{Responses from modeling}

In this case the crossing geometry from the drawing has been used. Several properties including contact forces, displacements and accelerations, stress/strain in rail material, sleeper and ballast are obtained, yet only the contact forces, crossing nose acceleration and Von Mises stress distribution are shown here. Figure 6a plots the contact forces in three directions, from which the impact on the crossing nose can be seen. The transition zone that from wing rail to crossing (facing direction) is also shown in the figure as the area where two red lines overlap. The acceleration of the crossing is obtained (Figure 6b) and compared with the ESAH-M measurement in different frequency bands, which shows good correlation with each other. For stress analysis since the profiles are changing along the crossing, stress/strain at different positions on the crossing are analyzed separately. The Von Mises stress distributions at four cross-sections are plotted in Figure 6c.

a

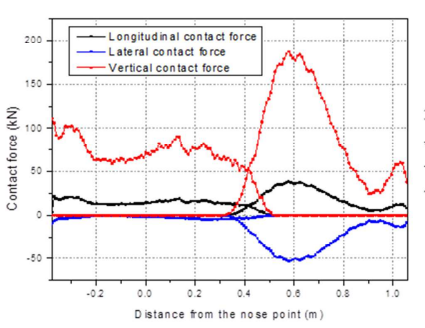

$\mathrm{b}$

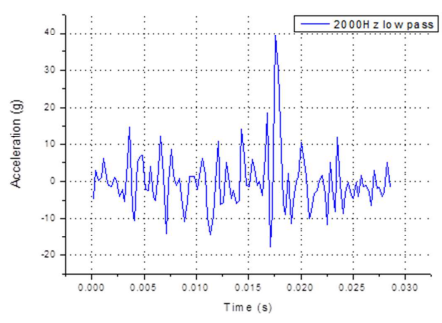

c

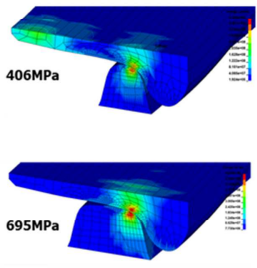

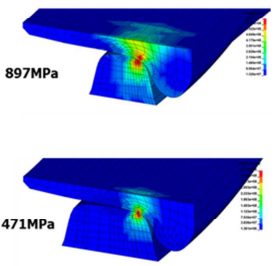

Figure 6. (a) contact forces, (b) Von Mises stress at different locations

Except for the properties shown in Figure 6, stress/strain results in railpad, sleeper and ballast are also available since they are modeled with solid elements. Parametric studies including variation of axle loads, velocities and friction coefficient as well as crossing geometry can be found in [8]. Moreover, analysis on facing and trailing directions can also be performed, in which case it is closely related to practical problems in the Dutch railway network.

\subsection{Fatigue life analysis}

It is known that in wheel/rail rolling contact problem the rails are subjected to multi-axial and non-proportional loadings. According to the critical plane approach stress types are also related to different types of damage, such as normal cracking and shear cracking. By the combined energy-density based and critical plane approach, crack plane can be determined and life cycles to fatigue crack initiation can be calculated.

Since the rail geometry is changing along the crossing, contact conditions such as contact angle, contact point and the contact radius are also changing. Therefore, stress/strain analysis as well as fatigue analysis of the crossing should be considered according to different positions. As long as the critical position is identified, the crack plane and fatigue life is then obtained at this position. This approach can be also applied to estimate different crossing designs. 


\section{FUTURE WORK}

As it is discussed above, by field measurements and finite element modeling the crossing performance can be analyzed. In order to improve the performance of the crossing, except for seeking more suitable crossing material, adjusting the crossing geometry (wing rail and crossing nose) as well as tuning the vertical elastic properties of the turnout can be performed.

Regarding to the crossing geometry, it is known that the height different between the wing rail and crossing determines the magnitude and location of the impact on the crossing. Therefore, the height of the wing rail can be tuned in FE model to reduce the impact on the crossing and wing rail in both traveling directions. On the other hand, the effects of the elastic properties of the other components (rail pads, under sleeper pads etc.) on the turnout performance can be analyzed using the 2-D finite element software DARTS_NL, wherein the wheel trajectory in the crossing obtained in the 3-D model will be used.

\section{CONCLUSION}

The approach for analyzing the crossing performance was discussed in this paper. Both experimental and numerical analyses were conducted to evaluate the dynamic behavior of the crossing and to further improve the crossing performance.

Field measurements including both geometry and acceleration measurements were performed on common single turnouts in the Dutch railway network. They were not only used for analyzing the measured crossing performance, but also providing the input for numerical modeling. The measured crossing geometry can be used to analyze the conditions of a crossing and to build up the numerical models of the crossing for different analyses such as model validation, problem diagnosis of a given crossing as well as the improvement of crossing geometry. The acceleration measurements indicated the fatigue areas in the crossing nose for each passing wheel and the crossing performance as well as the quality of wheels were assessed.

Meanwhile, a 3-D finite element model of a whole wheelset rolling over the crossing has been developed. The wing rail and crossing nose geometry as well as the wheel geometry have been used in the model. The dynamic simulations were performed using the explicit finite element analysis. The numerical responses of the model comprise the dynamic contact forces between the wheelset and the crossing, displacements of the wheelset and crossing as well as the local contact stress and strain distributions. Thus the impact of the wheel on the crossing was studied using this model and the fatigue life of the crossing was predicted.

By this procedure the crossing performance were assessed and further improvement of the crossing nose and wing rail geometry can be realized.

\section{REFERENCE}

[1] Pletz, M., Daves, W. Ossberger \& H. 2012. A wheel set/crossing model regarding impact, sliding and deformation-Explicit finite element approach, Wear, 294-295: 446-456.

[2] Markine, V.L, Steenbergen, M.J.M.M \& Shevtsov, I.Y. 2011. Combatting RCF on switch points by tuning elastic track properties, Wear, 271: 158-167.

[3] Wan, C., Markine, V.L \& Shevtsov, I.Y. 2014. Improvement of vehicle-turnout interaction by optimising the shape of crossing nose, Vehicle System Dynamics, Published online before print.

[4] Pålsson, B.A. 2014. Optimisation of Railway Switches and Crossings, PhD thesis, Chalmers University of Technology, ISBN 978-91-7385-978-3.

[5] Pletz, M., Daves, M., Yao, W., Ossberger, H. 2014. Rolling Contact Fatigue of three crossing nose materials-multi-scale FE approach, Wear, 314: 69-77.

[6] Norberg, L. Fatigue properties of austenitic Mn-steel in explosion depth hardened condition,, Master Thesis, Chalmers University of Technology.

[7] Xin, L. Markine, V.L. Shevtsov, I.Y. 2014. "Simulation of Railway Crossing Damage Due to Welding Defect", The Second International Conference on Railway Technology: Research, Development and Maintenance, (Civil-Comp Press, Ajaccio, France, 2014). 
[8] Xin, L. Markine, V.L. Shevtsov, I.Y. Numerical analysis of the dynamic interaction between wheelset and turnout crossing using explicit finite element method, submitted to Vehicle System Dynamics.

[9] Wan, C., Markine, V.L \& Shevtsov, I.Y. 2013. Analysis of train/turnout vertical interaction using a fast numerical model and validation of that model, Journal of Rail and Rapid Transit, 1-14.

[10] Pålsson, B.A. \& Nielsen, J.C.O. 2012. Wheel-rail interaction and damage in switches and crossings, Vehicle System Dynamics, 43-58.

[11] Markine, V.L, \& Shevtsov, I.Y. 2012. Experimental Analysis of the Dynamic Behaviour of Railway Turnouts," The Eleventh International Conference on Computational Structures Technology, Dubrovnik, Croatia.

[12] Markine, V.L, \& Shevtsov, I.Y. 2013. An Experimental Study on Crossing Nose Damage of Railway Turnout in the Netherlands, The Fourteenth International Conference on Civil, Structural and Environmental Engineering Computing(Civil-Comp Press, Cagliari, Italy).

[13] Xin, L. Markine, V.L. Shevtsov, I.Y. 2015. Numerical Analysis of Rolling Contact Fatigue Crack Initiation and Fatigue Life Prediction of the Railway Crossing, Proceedings of $10^{\text {th }}$ International Conference on Contact Mechanics, 2015.

[14] Xin, L. Markine, V.L. Shevtsov, I.Y. 2013. Analysis of the wheelset and turnout crossing interaction by multibody and finite element simulation, Proceedings of the Fifteen International Conference on Civil, Structural and Environmental Engineering Computing (Civil-Comp Press, Cagliari, Italy).

[15] Ringsberg, J. W., Bjarnehed, H., Johansson, A. \& Josefson, B. L. 2000. Rolling contact fatigue of rails- finite element modelling of residual stresses, strains and crack initiation. Journal of Rail and Rapid Transit, 214: 7-19. 